\title{
Kinetic Effects on Slowly Rotating Magnetic Islands in Tokamaks
}

\author{
Mattia Siccinio, Emanuele Poli \\ Max-Planck-Institut für Plasmaphysik, EURATOM Association \\ Boltzmannstrasse, 2 D-85748 \\ Garching bei München, Germany
}

June 19, 2009

\begin{abstract}
The current flowing around a magnetic island and connected to its rotation with respect to the plasma is studied by means of a drift-kinetic approach. It is shown that the current due to the change of the precession frequency of the trapped particles in the island potential can compete with the standard polarization current for island rotation frequencies close to or below the diamagnetic frequency. The passing particles are found on the contrary to have little impact on the standard picture. The analytical results are shown to explain the features of the island current seen in numerical simulations.
\end{abstract}

\section{Introduction}

The tearing mode is a non-ideal MHD instability which changes the topology of the magnetic field by reconnecting separate field lines, thus causing the appearance of magnetic islands. In a toroidal fusion device, this severely deteriorates the radial confinement of particles and energy. These modes are localized on so called resonant surfaces, where the safety factor $q$ is a rational number. First studies $[1,2]$ described the tearing mode as a dissipative phenomenon for the magnetic energy stored in the plasma, so that the growth rate of the mode was basically determined by the equilibrium magnetic configuration through the parameter $\Delta^{\prime}$. Later, it was shown both theoretically $[3,4]$ and experimentally $[5,6]$ that the tearing mode can also be destabilized in a tokamak by the loss of bootstrap current caused by the appearance of a "seed" island, and this neoclassical drive characterizes the so called neoclassical tearing modes (NTMs). While the neoclassical drive and the stabilizing effect of the equilibrium current profile can describe the nonlinear saturation of the mode under experimental conditions, to compute the stability and the growth of a seed island, one needs to take into account other effects which are still to be fully 
explained. Among these, the rotation of the island with respect to the plasma turns out to potentially play a major role. For "fast" rotating islands (i.e. $\omega$ sufficiently larger than $k_{\|} v_{\text {th }}$, where $\omega$ is the island rotation frequency with respect to the plasma, i.e. in the local equilibrium $\mathbf{E} \times \mathbf{B}$ frame, $k_{\|}$is the parallel wave vector defined in Eq. (6) below and $v_{\text {th }}$ is the ion thermal velocity) sufficiently larger than the ion banana width [7], a well-known contribution comes from the polarization current $[8,9]$, which arises because particles experience a time-dependent electric field during their streaming around the island. This phenomenon has different physical features depending on collisionality $[10,11,12,13]$, and frequency [14]. Moreover, other effects have been shown to be relevant, for example finite ion banana width effects $[7,15]$, or even finite Larmor radius effects [16]. As a matter of fact, for small islands the gyroradius scale starts to be relevant, and a gyro-kinetic approach can be necessary to get a satisfactory physical picture $[17,18,19]$.

As a general theory for the determination of the mode rotation frequency $\omega$ is currently not available, we perform a parametric study in $\omega$ focusing on a frequency range which has not been explored yet, even though it can be physically meaningful for a NTM. A common assumption in the theory of the polarization current consists in the ordering $\omega \sim \omega_{*}$, where $\omega_{*}$ is the electron diamagnetic frequency. According to the scaling criteria discussed in detail in Section 4, this ordering leads to $\omega \sim k_{\|} v_{\text {th }}$. Physically, this means that the island rotation occurs on the same timescale as the transit time of the particles around it, potentially leading to resonant interactions between the mode and the particles, as already pointed out previously [14]. To investigate these processes, a kinetic approach is necessary. In a previous study [11], focusing on the contribution of the polarization current, terms in $k_{\|} v_{\text {th }}$ have been disregarded. In this paper, we retain these terms and investigate their contribution to the currents generated by a rotating magnetic island. We neglect the mode evolution due to the plasma response, so that the island width and its rotation frequency are constant with time. Moreover, we assume for simplicity a flat pressure gradient for the background plasma. Although fundamental for a self-consistent determination of the island dynamics, the inclusion of a finite gradient in the background pressure is not strictly necessary to understand the phenomena related to the resonances between island and particle motion, which are the subject of the present paper.

We show that, at low frequencies, the contribution of the polarization current can be overcome by other electric and magnetic effects. In particular, we demonstrate in this work that a crucial contribution comes from the mutual interaction between the island propagation and the electric and magnetic precession of the trapped particles in toroidal direction. The corresponding perturbed parallel current is shown to stabilize the NTM for $\omega>0$, or for $|\omega|$ sufficiently larger than $\omega_{\text {tp }}$ if $\omega<0$, where $\omega_{\text {tp }}$ is the precession frequency of trapped particles defined in Eq. (29) below. Passing particles are on the contrary shown to give a negligible current contribution. The analytical results are shown to explain the behaviour of the island current as calculated by means of 
the Hamiltonian drift-kinetic code HAGIS [20].

This paper is structured as follows. In Section 2, the chosen magnetic geometry is outlined. In Section 3 we describe the analytical and numerical techniques adopted to solve the drift-kinetic equation. In Section 4 and 5 we solve the drift-kinetic equation in the $\omega \sim k_{\|} v_{\text {th }}$ and in the $\omega \ll k_{\|} v_{\text {th }}$ regime. This allows us to highlight the role of passing and trapped particles, respectively. Analytic solutions are compared to numerical simulations. The results are further discussed and summarized in Section 6.

\section{Magnetic Geometry}

In the calculations which follow, we assume an axisymmetric, large-aspect-ratio toroidal geometry, with circular cross section. We define $\epsilon$ as the inverse aspect ratio. For convenience we use here the same notation as in Ref. [11]. The coordinate system is represented by a poloidal flux $\chi$, which can be used as a radial coordinate, a toroidal angle $\zeta$ and a poloidal angle $\theta$. This choice is such that

$$
\nabla \zeta \times \nabla \chi=r B_{\theta} \nabla \theta,
$$

where $r$ is the minor radius coordinate and $B_{\theta}$ the poloidal component of the magnetic field. The equilibrium magnetic field is expressed by

$$
\mathbf{B}=I(\chi) \nabla \zeta+\nabla \zeta \times \nabla \chi,
$$

having defined $I(\chi)=R B_{\zeta}$, where $R$ is the major radius and $B_{\zeta}$ the toroidal component of the magnetic field. Thus, the equilibrium magnetic field is such that $\mathbf{B} \cdot \nabla \chi=0$. We suppose that the magnitude of the magnetic field $B$ varies over the poloidal cross section as $B=B_{0}(1-\epsilon \cos \theta)$. The helical angle $\xi$ is introduced as:

$$
\xi=m \theta-n \zeta-\omega t,
$$

where $m, n$ are the poloidal and toroidal mode number, respectively, and $\omega$ is the island rotation frequency, supposed constant in time for simplicity. We can include the magnetic island as a perturbation in the magnetic field writing

$$
\mathbf{B}=I(\chi) \nabla \zeta+\nabla \zeta \times \nabla(\chi+\psi),
$$

where

$$
\psi=\tilde{\psi} \cos \xi,
$$

where $\tilde{\psi}$ is supposed to be a constant according to the well-known constant- $\psi$ approximation [2]. We can define a new coordinate system, using $\chi, \xi$ and $\theta$, and this is the system which will be used in this article from here on. It is possible to build a perturbed flux surface label $\Omega$, such that in presence of the magnetic island $\mathbf{B} \cdot \nabla \Omega=0$. Writing with a subscript $s$ quantities which are calculated on the resonant surface, we obtain 


$$
\Omega=\frac{2\left(\chi-\chi_{s}\right)^{2}}{W_{\chi}^{2}}-\cos \xi
$$

where $W_{\chi}^{2}=4 \tilde{\psi} q_{s} / q_{s}^{\prime}$, is connected to the island half-width $w$ by the relation $W_{\chi}=R B_{\theta} w, q$ is the safety factor and the apex ' refers to the derivative with respect to $\chi$. With the help of the function $\Omega$, one arrives at the following expression for the parallel gradient:

$$
\nabla_{\|}=\frac{1}{R q} \frac{\partial}{\partial \theta}+\left.k_{\|} \frac{\partial}{\partial \xi}\right|_{\Omega}
$$

where

$$
k_{\|}=-m \frac{\left(\chi-\chi_{s}\right)}{R q} \frac{q_{s}^{\prime}}{q_{s}} .
$$

It can be seen that $\psi$ can be thought as a perturbation of the parallel component of the magnetic vector potential A. As we suppose that every parallel electric field $E_{\|}$is immediately shorted out by the very fast electron streaming along the field lines, we can obtain an analytical expression for the $(\theta$-independent) scalar potential using the Faraday's law:

$$
\nabla_{\|} \Phi=-\frac{1}{c} \frac{\partial A_{\|}}{\partial t} \Rightarrow \Phi=\frac{\omega q}{m c}\left[\chi-\chi_{s}-h(\Omega)\right],
$$

where use has been made of Eq. (26) below. Here, $h(\Omega)$ is a function which plays the role of an integration constant, and can be determined from boundary conditions. A simple choice for $h(\Omega)$ can be found in Ref. [10]:

$$
h(\Omega)=\frac{W_{\chi}}{\sqrt{2}}(\sqrt{\Omega}-1) \Theta(\Omega-1),
$$

where $W_{\chi}$ is defined to have the same sign as $\left(\chi-\chi_{s}\right)$, and $\Theta(x)$ is the Heaviside step function. This choice allows the scalar potential to vanish far away from the magnetic island. Moreover, this fixes $h(\Omega)$ to the value of zero inside the island ( $\Omega=-1$ corresponds to the O-point, while $\Omega=1$ to the island separatrix).

\section{The Drift-Kinetic Equation}

\subsection{Analytic Approach}

The drift-kinetic equation describes the time evolution of the distribution of the particles' guiding centres in a magnetized plasma. This means that particles are treated like streaming along magnetic field lines through their parallel velocity, and drifting across the field lines through their electric and magnetic drifts. Gyration motion is implicitly present in the mirror force and in defining the magnetic moment $\mu$ (supposed to be a constant), but spatial variation of physical quantities on the Larmor radius scale are neglected: 


$$
\frac{\partial f_{j}}{\partial t}+v_{\| j} \nabla_{\|} f_{j}+\mathbf{v}_{\mathbf{E} \times \mathbf{B}} \cdot \nabla f_{j}+\mathbf{v}_{D j} \cdot \nabla f_{j}-\frac{q_{j}}{m_{j}} \frac{\mathbf{v}_{D j} \cdot \nabla \Phi}{v} \frac{\partial f_{j}}{\partial v}=C_{j}\left(f_{j}\right) .
$$

The subscript $j$ labels the different species that are present in the plasma. Spatial derivatives have to be taken at constant kinetic energy. This form of the drift kinetic equation is explained in detail in Ref. [21].

The magnetic drift velocity can be written in the form

$$
\mathbf{v}_{D j}=-v_{\| j} \mathbf{b} \times \nabla\left(\frac{v_{\| j}}{\omega_{c j}}\right),
$$

where $\mathbf{b}$ is the unit vector parallel to the magnetic field and $\omega_{c j}$ is the cyclotron frequency for the $j$-th particle species. In this work we are focusing on ions, supposing that the only contribution of electrons is shorting out the parallel electric field $E_{\|}$. As every physical quantity such as temperature $(T)$ or the distribution itself is referred to ions, we can from here on drop the subscript $j$ without ambiguity. We keep the index $i$ only for the ion mass $m_{i}$ and for the ion charge $q_{i}$ in order to avoid confusions with the poloidal mode number $m$ and the safety factor $q$, respectively. Eq. (8) is solved splitting the distribution function into an analytically known part $F_{0}$, assumed here to be an isotropic Maxwellian

$$
F_{M}(v)=n_{0}\left(\frac{m_{i}}{2 \pi T}\right)^{3 / 2} e^{m_{i} v^{2} / 2 T}
$$

(where the density $n_{0}$ and the temperature $T$ are assumed to be uniform, (f. Introduction), and a part $g$ do be determined perturbatively. Specifically, in the coordinate system discussed in Section 2, writing the full expression for drifts, Eq. (8) takes the form

$$
\begin{array}{r}
-\omega \frac{\partial g}{\partial \xi}+\frac{v_{\|}}{R q} \frac{\partial g}{\partial \theta}+\left.k_{\|} v_{\|} \frac{\partial g}{\partial \xi}\right|_{\Omega}+m \frac{c}{B} \frac{I}{R q} \frac{\partial \Phi}{\partial \chi} \frac{\partial g}{\partial \xi}+ \\
-m \frac{c}{B} \frac{I}{R q} \frac{\partial \Phi}{\partial \xi} \frac{\partial g}{\partial \chi}+\frac{I v_{\|}}{R q} \frac{\partial}{\partial \theta}\left(\frac{v_{\|}}{\omega_{c}}\right) \frac{\partial g}{\partial \chi}-m \frac{I v_{\|}}{R q} \frac{\partial}{\partial \chi}\left(\frac{v_{\|}}{\omega_{c}}\right) \frac{\partial g}{\partial \xi} \\
-\frac{q_{i}}{m_{i}}\left[\frac{I v_{\|}}{R q} \frac{\partial}{\partial \theta}\left(\frac{v_{\|}}{\omega_{c}}\right) \frac{\partial \Phi}{\partial \chi}-m \frac{I v_{\|}}{R q} \frac{\partial}{\partial \chi}\left(\frac{v_{\|}}{\omega_{c}}\right) \frac{\partial \Phi}{\partial \xi}\right] \frac{\partial g}{\partial v}= \\
=-\frac{q_{i} F_{M}}{T}\left[\frac{I v_{\|}}{R q} \frac{\partial}{\partial \theta}\left(\frac{v_{\|}}{\omega_{c}}\right) \frac{\partial \Phi}{\partial \chi}-m \frac{I v_{\|}}{R q} \frac{\partial}{\partial \chi}\left(\frac{v_{\|}}{\omega_{c}}\right) \frac{\partial \Phi}{\partial \xi}\right],
\end{array}
$$

where we have neglected the collision operator (the role of collisions will be discussed later on). The parallel velocity $v_{\|}$is written in the so called pitchangle variables [21]:

$$
v_{\|}=\sigma v \sqrt{1-\lambda B} .
$$

Here, $\lambda=2 \mu / m_{i} v^{2}$, where $\mu$ is the magnetic moment, and $\sigma$ is the sign of the parallel velocity. A trapped particle has $1 / B>\lambda>1 / B_{M}$, where $B_{M}$ is 
the maximum value of the magnetic field on a given flux surface. With these variables, the integration operator over velocity space becomes

$$
\int_{-\infty}^{\infty} \ldots \mathrm{d}^{3} v=\pi B \sum_{\sigma= \pm 1} \int_{0}^{\infty} v^{2} \mathrm{~d} v \int_{0}^{\frac{1}{B}} \cdots \frac{\mathrm{d} \lambda}{\sqrt{1-\lambda B}}
$$

Eq. (10) is solved performing a double parameter expansion of the perturbed distribution $g$, i. e.

$$
g=\sum_{m, n}^{\infty} g^{(m, n)} \delta^{m} \Delta^{n}
$$

where

$$
\delta=\frac{\rho_{b}}{w} \quad \Delta=\frac{w}{a}
$$

are supposed to be two small parameters (here, $\rho_{b}$ is the ion banana width and $a$ the tokamak minor radius). The ordering of the various terms in Eq. (10) with respect to $\delta$ and $\Delta$ will be discussed in Section 4 and 5 . The only "free" parameter is the island propagation frequency $\omega$. In Section 4 we mantain the ordering assumptions for $\omega$ used in Ref. [11], which leads to $\omega \sim k_{\|} v_{\text {th }}$. In Section 5 , we order $\omega \sim \omega_{D}$, where

$$
\omega_{D}=\frac{q}{\operatorname{Rr} \omega_{c}} \frac{1}{2 \theta_{b}} \int_{-\theta_{b}}^{\theta_{b}}\left[\frac{\mu B}{m_{i}}+v_{\|}^{2}\right] \cos \theta \mathrm{d} \theta
$$

is the (bounce averaged) toroidal precession frequency of the trapped particles [22], defining as $\theta_{b}$ the bounce angle.

The aim of these ordering assumptions is to study which physical phenomena can take place when the island propagation frequency $\omega$ starts to be comparable with the particles' transit frequencies around the island itself, which are basically linked to $k_{\|} v_{\|}$(with $v_{\|} \sim v_{\text {th }}$ ) for passing particles, and to $\omega_{D}$ for trapped particles. This second case can also be thought as ordering the island propagation frequency like the parallel streaming of the trapped particle, as $\omega \sim \omega_{D}$ means $\omega \sim \sqrt{\epsilon} k_{\|} v_{\text {th }}$. This point will be developed in Section 5 .

\subsection{Numerical Approach}

The HAGIS code (HAmiltonian GuIding centre System) [20] solves the drift kinetic equation for ions by means of a Hamiltonan approach and with the $\delta f$ technique. This method consists in writing the distribution $f$ as $f=F_{0}+\delta f$, where $F_{0}$ is known and arbitrary. This approach is completely general, but can be convenient from the numerical point of view only if $f$ is not expected to be so different from the chosen $F_{0}$, that means

$$
\frac{\delta f}{F_{0}} \ll 1,
$$


which is what we expect to find. The HAGIS code calculates the evolution in time of the distribution function by means of "markers" which span the whole phase space and represent the ions. These "markers" evolve according to the Hamiltonian equations of motion, which are integrated by the code. As in Section 3.1, we choose as $F_{0}$ a space-independent isotropic Maxwellian. In the simulations presented in the next Sections, we consider a tokamak with circular concentric flux surfaces and major radius $R=8 \mathrm{~m}$, aspect ratio $a / R=0.5$, magnetic field $B_{0}=8 \mathrm{~T}$, deuterium plasma with density $n_{i}=10^{20} \mathrm{~m}^{-3}$ and temperature $T=5 \mathrm{keV}$. Collisions are described by a Monte Carlo algorithm which models pitch-angle scattering [23]. A $(m=3, n=2)$ magnetic island with a fixed half-width $w=6.8 \mathrm{~cm}$ is included in the simulations, and the island frequency $\omega$ is treated as an input parameter. With these values, the ratio between the island width and the thermal ion banana width corresponds to $w / \rho_{b} \approx 9.6$ and the ratio between the island width and the tokamak minor radius $a$ corresponds to $w / a \approx 0.017$. The space domain is divided into "radial" cells (between two neighbouring perturbed flux surfaces) and into helical cells, in such a way that the volume between two X-points of a magnetic island consists of six helical cells (for further details see Ref. [24]). All numerical results presented below refer to the "upper" half of the magnetic island (i.e. from O-point to X-point travelling in the positive- $\theta$ direction). In the "lower" half, results can be shown to simply change their sign.

\section{The $\omega \sim k_{\|} v_{\|}$Regime}

\subsection{Solution of The Drift-Kinetic Equation}

The solution of the drift-kinetic equation presented in this paper follows closely the analytic treatment presented in Ref. [11]. In that paper, $F_{0}$ is chosen so that an adiabatic response of the ions to the electrostatic potential is included, and equilibrium gradients are retained. Under these circumstances, the lowest order perturbation $g^{(0,0)}$ has the double role of cancelling the adiabatic response and of introducing the perturbations due to the island in the density and temperature profiles. The next-order term in the expansion of the distribution

function with respect to $\delta$, i.e. $g^{(1,0)}$, is shown to be linked to the neoclassical parallel fluxes, included that due to radial electric fields. The frequency $\omega_{E}$, corresponding to the latter, is defined as in Ref. [25]:

$$
\omega_{E}=c \frac{E_{r}}{R B_{\theta}},
$$

where in this case the radial electric field we are interested in is generated by the island itself. By means of the charge continuity equation, Wilson et al. show that this electric drift is the physical trigger of the polarization current, because it allows trapped particles to experience a time-varying electric field.

Let us now turn to Eq.(10). In this Section, we follow the ordering assumptions adopted in Ref. [11]. This in particular consists in 


$$
\frac{\partial}{\partial \theta} \sim \frac{\partial}{\partial \xi} \sim 1 \quad \frac{q_{i} \Phi}{T} \sim \frac{g}{F_{M}} \sim \Delta \quad \rho_{\theta} \sim w
$$

( $\rho_{\theta}$ being the poloidal ion Larmor radius). As for the island propagation frequency, if the pressure profile possesses a finite gradient characterized by the length $L_{p}$, a natural choice is to assume $\omega$ to be of the same order as the diamagnetic frequency $\left(\omega_{*}=m c T n_{e}^{\prime} / q_{i} q n_{e}\right.$, with $n_{e}^{\prime}=\partial n_{e} / \partial \chi$, being $n_{e}$ the electron density). Supposing $n_{e}^{\prime} \sim n_{e} / R B_{\theta} a$ (i. e. making the choice $L_{p} \sim a$ ), this implies (cf. Eq. (13)):

$$
\frac{R q}{v_{\|}} \omega \sim \frac{R q}{v_{\|}} \omega_{*} \sim \frac{R q}{v_{\mathrm{th}}} \frac{m c T n_{e}^{\prime}}{q_{i} q n_{e}} \sim \frac{\rho_{\theta}}{a} \sim \Delta .
$$

The term describing the parallel streaming turns out to be of the same order, since (see Eq. (6))

$$
\frac{R q}{v_{\|}} k_{\|} v_{\|} \sim\left(\chi-\chi_{s}\right) \frac{q_{s}^{\prime}}{q_{s}} \sim \frac{w}{r} \sim \Delta,
$$

having assumed $\left(\chi-\chi_{s}\right) \sim W_{\chi}$, as we focus our calculations on the vicinity of the island, and $L_{p} \sim L_{q} \sim \epsilon L_{s}, L_{q}$ and $L_{s}$ being the radial scale length of the safety factor and of the magnetic shear, respectively.

As already mentioned, to point out the effects linked to the mutual rotation of particles and island, in our analysis we take a flat pressure profile, $\omega_{*}=0$, and treat $\omega$ as a free parameter. In this Section, we retain the ordering $\omega R q / v_{\|} \sim \Delta$, which implies $\omega \sim k_{\|} v_{\text {th }}$. The resulting ordering of the terms in Eq. (10) is

$$
\Delta: 1: \Delta: \Delta: \Delta: \delta: \Delta \delta: \Delta \delta: \Delta^{2} \delta=\delta: \Delta \delta .
$$

It is worthwile to stress the fact that the term $\mathbf{v}_{D} \cdot \nabla$ in Eq. (8) consists in two different components which, under our assumptions, are not of the same order.

We now turn to the order-by-order solution of Eq.(10). As we do not include an adiabatic split in $F_{0}$, and as we do not consider density and temperature equilibrium gradients, according to the meaning of $g^{(0,0)}$ elucidated above, we can assume

$$
g^{(0,0)}=0
$$

The $\mathcal{O}(\delta)$ equation reads

$$
\frac{v_{\|}}{R q} \frac{\partial}{\partial \theta} g^{(1,0)}=-\frac{q_{i} F_{M}}{T} \frac{I v_{\|}}{R q} \frac{\partial}{\partial \theta}\left(\frac{v_{\|}}{\omega_{c}}\right) \frac{\partial \Phi}{\partial \chi},
$$

which can be directly integrated to give

$$
g^{(1,0)}=-I \frac{v_{\|}}{\omega_{c}} \frac{\partial \Phi}{\partial \chi} \frac{q_{i} F_{M}}{T}+\bar{h}_{P}^{(1,0)}+\bar{h}_{T}^{(1,0)}
$$

(in the first term on the right-hand side of this expression, it is easy to identify the first-order expansion of a Maxwellian shifted by a velocity $c E_{r} / B_{\theta}$ in the 
parallel direction). The bar over a function indicates that it is $\theta$-independent, so, $\bar{h}$-functions represent the integration constants. We have divided them, separating the passing region of phase space (subscript $P$ ) from the trapped one (subscript $T$ ).

As $g^{(0,0)}=0$, the $\mathcal{O}(\Delta)$ equation simply amounts to

$$
\frac{v_{\|}}{R q} \frac{\partial}{\partial \theta} g^{(0,1)}=0
$$

The $g^{(0,1)}=\bar{g}^{(0,1)}$ is $\theta$-independent. This information allows us to eliminate the contribution of $g^{(0,1)}$ when performing the $\theta$ averages in Eq. (21) below.

To calculate the $\bar{h}$-functions, we move to the $\mathcal{O}(\Delta \delta)$ equation. Using the identity

$$
\frac{\partial}{\partial t}+c \frac{\mathbf{B} \times \nabla \Phi}{B^{2}} \cdot \nabla=\frac{\mathrm{d}_{0}}{\mathrm{~d} t}=\left.\frac{\mathrm{d} h}{\mathrm{~d} \Omega} \frac{\omega}{m \tilde{\psi}} R q k_{\|} \frac{\partial}{\partial \xi}\right|_{\Omega}+\frac{\omega}{m}\left(1-\frac{\partial h}{\partial \chi}\right) \frac{\partial}{\partial \theta},
$$

we obtain

$$
\begin{array}{r}
\left.\frac{\mathrm{d} h}{\mathrm{~d} \Omega} \frac{\omega}{m \tilde{\psi}} R q k_{\|} \frac{\partial g^{(1,0)}}{\partial \xi}\right|_{\Omega}+\frac{\omega}{m}\left(1-\frac{\partial h}{\partial \chi}\right) \frac{\partial g^{(1,0)}}{\partial \theta}+\frac{v_{\|}}{R q} \frac{\partial g^{(1,1)}}{\partial \theta}+ \\
+\left.k_{\|} v_{\|} \frac{\partial g^{(1,0)}}{\partial \xi}\right|_{\Omega}+\frac{I v_{\|}}{R q} \frac{\partial}{\partial \theta}\left(\frac{v_{\|}}{\omega_{c}}\right) \frac{\partial g^{(0,1)}}{\partial \chi}=m \frac{I v_{\|}}{R q} \frac{\partial}{\partial \chi}\left(\frac{v_{\|}}{\omega_{c}}\right) \frac{\partial \Phi}{\partial \xi} \frac{q_{i} F_{M}}{T} .
\end{array}
$$

In order to calculate the $\bar{h}$-functions, it is convenient to use the bounce average operator, which has two different definitions in the trapped and in the passing region of phase space. We consider the passing region first. Starting from the average operator

$$
\langle\ldots\rangle_{\theta}=\frac{1}{2 \pi} \oint \ldots \mathrm{d} \theta
$$

the bounce average operator is defined as [21]

$$
\left\langle\frac{R q}{v_{\|}} \ldots\right\rangle_{\theta}=\frac{1}{2 \pi} \oint \frac{R q}{v_{\|}} \ldots \mathrm{d} \theta .
$$

So we find

$$
\begin{array}{r}
\left.\left\langle\frac{\mathrm{d} h}{\mathrm{~d} \Omega} \frac{\omega}{m \tilde{\psi}} \frac{R q k_{\|}}{v_{\|}}+k_{\|}\right\rangle_{\theta} \frac{\partial \bar{h}_{P}^{(1,0)}}{\partial \xi}\right|_{\Omega}= \\
\left.I\left\langle\left(\frac{\mathrm{d} h}{\mathrm{~d} \Omega} \frac{\omega}{m \tilde{\psi}} \frac{R q k_{\|}}{v_{\|}}+k_{\|}\right) \frac{v_{\|}}{\omega_{c}}\right\rangle_{\theta} \frac{\partial}{\partial \xi}\right|_{\Omega}\left(\frac{\partial \Phi}{\partial \chi}\right) \frac{q_{i} F_{M}}{T}+ \\
I\left\langle\frac{m}{R q} \frac{\partial}{\partial \chi}\left(\frac{v_{\|}}{\omega_{c}}\right)\right\rangle_{\theta} \frac{\partial \Phi}{\partial \xi} \frac{q_{i} F_{M}}{T} .
\end{array}
$$


As we focus on the contributions which vanish on the flux-surface average, $\bar{h}$ functions are supposed here to vanish when averaged over $\Omega$, where the average on $\Omega$ is defined as

$$
\langle\ldots\rangle_{\Omega}=\frac{\oint \cdots \mathrm{d} \xi / \sqrt{\Omega+\cos \xi}}{\oint \mathrm{d} \xi / \sqrt{\Omega+\cos \xi}} .
$$

This is because all the contributions which do not vanish by using this operator are related to the bootstrap current. So we obtain

$$
\begin{aligned}
& \bar{h}_{P}^{(1,0)}=-\frac{4 I}{W_{\chi}^{2}} \frac{\omega q}{m c} \frac{\mathrm{d} h}{\mathrm{~d} \Omega} \frac{q_{i} F_{M}}{T}\left\langle\left(\frac{\mathrm{d} h}{\mathrm{~d} \Omega} \frac{\omega}{m \tilde{\psi}} \frac{R q}{v_{\|}}+1\right) \frac{v_{\|}}{\omega_{c}}+\frac{q_{s}}{q_{s}^{\prime}} \frac{\partial}{\partial \chi}\left(\frac{v_{\|}}{\omega_{c}}\right)\right\rangle_{\theta} . \\
& {\left[\left\langle\frac{\mathrm{d} h}{\mathrm{~d} \Omega} \frac{\omega}{m \tilde{\psi}} \frac{R q}{v_{\|}}+1\right\rangle_{\theta}\right]^{-1}\left[\chi-\langle\chi\rangle_{\Omega}\right] .}
\end{aligned}
$$

The physical features related to this solution will be discussed in the next Section.

We now solve Eq. (21) in the trapped region of velocity space. The corresponding bounce average operator becomes [21]

$$
\left\langle\frac{R q}{\left|v_{\|}\right|} \ldots\right\rangle_{\theta}^{T}=\sum_{\sigma= \pm 1} \frac{1}{2 \theta_{b}} \int_{-\theta_{b}}^{\theta_{b}} \frac{R q}{\left|v_{\|}\right|} \ldots \mathrm{d} \theta
$$

yielding

$$
\begin{aligned}
\left.\frac{\partial \bar{h}_{T}^{(1,0)}}{\partial \xi}\right|_{\Omega} & =I\left\langle\left. k_{\|} \frac{\left|v_{\|}\right|}{\omega_{c}} \frac{\partial}{\partial \xi}\right|_{\Omega}\left(\frac{\partial \Phi}{\partial \chi}\right)+m \frac{\partial}{\partial \chi}\left(\frac{\left|v_{\|}\right|}{\omega_{c}}\right) \frac{\partial \Phi}{\partial \xi}\right\rangle_{\theta}^{T} \\
& \times\left(\left\langle\frac{\mathrm{d} h}{\mathrm{~d} \Omega} \frac{\omega}{m \tilde{\psi}} \frac{R q k_{\|}}{\left|v_{\|}\right|}\right\rangle_{\theta}^{T}\right)^{-1} \frac{q_{i} F_{M}}{T} .
\end{aligned}
$$

We can approximate the right-hand side of this equation noting that

$$
\left\langle\frac{1}{v_{\|}}\right\rangle_{\theta}^{-1}\left\langle\nabla v_{\|}\right\rangle_{\theta}=\left\langle v_{\|} \nabla v_{\|}\right\rangle_{\theta}+\mathcal{O}\left(\epsilon^{2}\right) .
$$

Eq. (25) and the identity

$$
\left.k_{\|} \frac{\partial \chi}{\partial \xi}\right|_{\Omega}=\frac{m}{q} \frac{\partial A_{\|}}{\partial \xi}=\frac{m}{q} \frac{\tilde{\psi}}{R} \sin \xi
$$

allow us to write Eq.(24) in the more perspicuous form

$$
\bar{h}_{T}^{(1,0)}=-\frac{q_{i} F_{M}}{T}\left[\omega_{D}+\omega_{\hat{s}}\right] \frac{\left[\chi-\langle\chi\rangle_{\Omega}\right]}{c} .
$$


Here, $\omega_{D}$ is defined as in Eq. (14) and $\omega_{\hat{s}}$ is related to the toroidal precession a of a trapped particle due to the magnetic shear [22],

$$
\omega_{\hat{s}}=\left\langle\frac{q \hat{s} v_{\|}^{2}}{r^{2} \omega_{c}}\right\rangle_{\theta}^{T},
$$

where $\hat{s}$ is the magnetic shear (the correlation of the $\omega_{D}$ and $\omega_{\hat{s}}$-terms with the poloidal component of magnetic drifts and the terms in $k_{\|} v_{\|}$, respectively, is discussed in Appendix I). As both $\omega_{D}$ and $\omega_{\hat{s}}$ are related to magnetic effects, from here on we define the magnetic toroidal precession frequency [22]:

$$
\omega_{\mathrm{tp}}=\omega_{D}+\omega_{\hat{s}}
$$

Note that, within this ordering, $\bar{h}_{T}^{(1,0)}$ does not depend on any quantity related to the island, apart from the average radial position $\langle\chi\rangle_{\Omega}$.

\subsection{The Perturbed Current}

The quasi-neutrality condition $\nabla \cdot \mathbf{J}=0$ is now used to derive an expression for the $\theta$-independent parallel current which closes the polarization drift. In Ref. [11], this equation reads

$$
\left.k_{\|} \frac{\partial J_{\|}^{\mathrm{Pol}}}{\partial \xi}\right|_{\Omega}=-\frac{q_{i} I}{\omega_{c}} \frac{\omega}{m \tilde{\psi}} \int \mathrm{d}^{3} v v_{\|} \frac{\partial}{\partial \chi}\left[\frac{\mathrm{d} h}{\mathrm{~d} \Omega}\left\langle\left. R q k_{\|} \frac{\partial g^{(1,0)}}{\partial \xi}\right|_{\Omega}\right\rangle_{\theta}\right]
$$

where the superscript Pol refers to the polarization current. Considering the physical relation previously discussed between $g^{(1,0)}$ and $\omega_{E}$, the integral on the right-hand side can be connected to the total time derivative of the electric precession (i.e. the acceleration along the island surface which allows the particle to experience a time-dependent electric field, see Eq.(20)). This is the physical mechanism of the neoclassical polarization. In the limit $\omega \gg k_{\|} v_{\|}$adopted in Ref. [11] (which is an assumption that goes beyond the chosen scaling, and was adopted to isolate the polarization current), $\bar{h}_{T}^{(1,0)}$ is zero, while a leading order annihilation takes place because the $\theta$-dependent part of $g^{(1,0)}$ and $\bar{h}_{P}^{(1,0)}$ differ only for higher order terms in $\epsilon$. As a result, the polarization current contributing to the island dynamics is carried only by the trapped particles and is therefore $\mathcal{O}\left(\epsilon^{3 / 2}\right)$.

In our case, the quasi-neutrality condition includes the terms in $k_{\|} v_{\|}$and has the following expression:

$$
\left.k_{\|} \frac{\partial J_{\|}}{\partial \xi}\right|_{\Omega}=-\frac{q_{i} I}{\omega_{c}} \frac{\partial}{\partial \chi} \int \mathrm{d}^{3} v v_{\|}\left\langle\left.\left[\frac{\mathrm{d} h}{\mathrm{~d} \Omega} \frac{\omega}{m \tilde{\psi}} R q k_{\|}+k_{\|} v_{\|}\right] \frac{\partial g^{(1,0)}}{\partial \xi}\right|_{\Omega}\right\rangle_{\theta} .
$$

The terms in square brackets represent the advection along the magnetic surface, which includes now, in addition to that due to the island rotation and to 
the electric drift $\mathrm{d}_{0} / \mathrm{d} t$ (see Eq. (20)), also the parallel streaming of the ions through the term in $k_{\|} v_{\|}$. According to Eq. (18) and (23), we obtain

$$
\begin{aligned}
& \left.\frac{\partial J_{\|}}{\partial \xi}\right|_{\Omega}=-\frac{q_{i} I}{\omega_{c}} \frac{\partial}{\partial \chi} \int \mathrm{d}^{3} v v_{\|} \Lambda F_{M}\left\langle( \overline { u } + v _ { \| } ) \cdot \left[\left(\frac{v_{\|}}{\omega_{c}}\right)+\right.\right. \\
& \left.\left.-\left\langle\left(\frac{\bar{u}}{v_{\|}}+1\right) \frac{v_{\|}}{\omega_{c}}+\frac{q_{s}}{q_{s}^{\prime}} \frac{\partial}{\partial \chi}\left(\frac{v_{\|}}{\omega_{c}}\right)\right\rangle_{\theta} \cdot\left\langle\frac{\bar{u}}{v_{\|}}+1\right\rangle_{\theta}^{-1}\right]\right\rangle_{\theta},
\end{aligned}
$$

with

$$
\Lambda=\left.\frac{4 I}{W_{\chi}^{2}} \frac{\omega q}{m c} \frac{\mathrm{d} h}{\mathrm{~d} \Omega} \frac{q_{i}}{T} \frac{\partial \chi}{\partial \xi}\right|_{\Omega}
$$

and

$$
\bar{u}=\frac{\mathrm{d} h}{\mathrm{~d} \Omega} \frac{\omega}{m \tilde{\psi}} R q .
$$

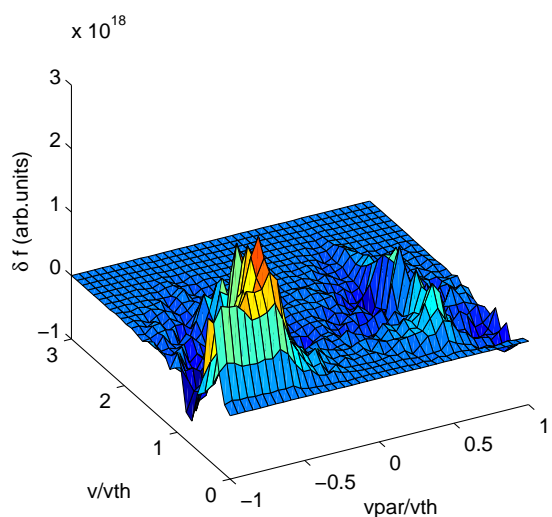

b)

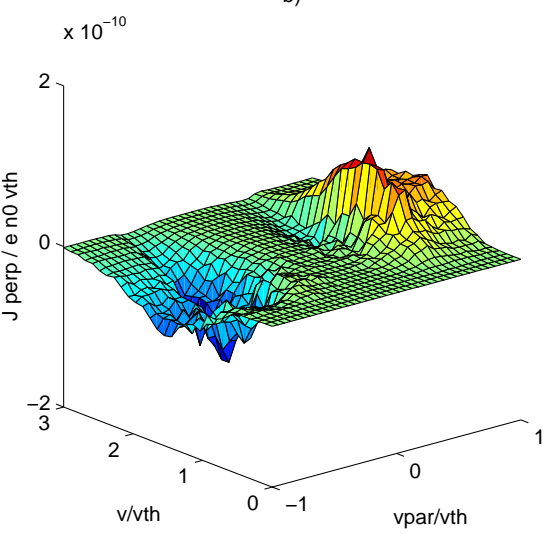

Figure 1: Perturbed distribution (a) and perpendicular current (b) in velocity space for the parameters described in Section 3.2 and $\omega=-4000 \mathrm{rad} / \mathrm{s}$, calculated at the fourth radial cells outside the magnetic island towards the centre (cf. Fig. 2).

Eq. (32) exhibits an important feature: the resonant denominator that appears in the distribution function (23) is almost cancelled by a similar numerator which arises from including the parallel streaming in the advection, as discussed above (this cancellation can be shown to occur up to $\mathcal{O}\left(\epsilon^{2}\right)$ ). This is a consequence of the motion of the particles, which have (at least in this ordering) vanishing $\theta$-averaged drifts across the flux surfaces when they approach the resonance.

Numerically, a resonant behaviour of the perturbed distribution function in velocity space can be clearly observed in Fig. 1a, which shows $\delta f$ in the vicinity of the inner island separatrix as a function of $v_{\|} / v$ and of $v / v_{\mathrm{th}}$. The resonance is visible in the part of the velocity space with $v_{\|}<0$. Passing particles with 
$v_{\|}>0$ resonate on the other (outer) side of the island, since $k_{\|}$reverses its sign across the resonant surface. The corresponding current (Fig. 1b), however, does not present such a resonant behaviour. Moreover, it shows an odd symmetry with respect to $v_{\|} / v$, so that the passing particles do not contribute to the total current, i. e. their contribution vanishes after integration over velocity space because of the sum over $\sigma$, as can be seen in Fig. 2 .

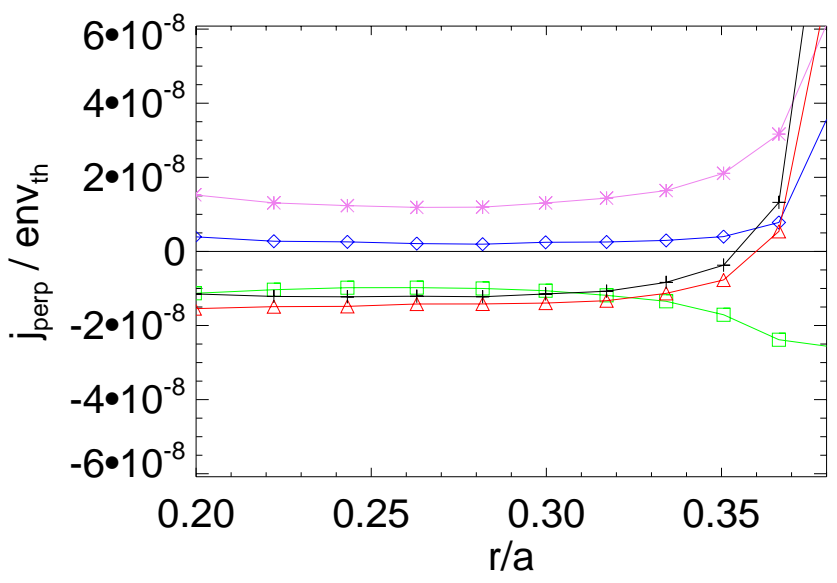

Figure 2: Radial profile of the perpendicular current (the inner island separatrix is located at $r / a=0.38$ ) for $\omega=-4000 \mathrm{rad} / \mathrm{s}$. Magenta stars represents the contribution of the passing particles with $v_{\|}>0$, green squares passing particles with $v_{\|}<0$, blue diamonds the total current due to passing particles, red triangles the trapped particles and the crossed black line the total perpendicular current. The sudden rise of the current close the island separatrix is due to the onset of the standard polarization current [14].

Formally, the contribution of the resonance to higher-order corrections (not retained in our calculations) should be treated employing an approach similar to that of e. g. Refs. [26, 27, 28, 17, 29]. However, we stress the fact that the Landau resonance contributes only to the "out of phase" part of the current, determining the island rotation. These effects are not included in the analysis presented in this paper, where the rotation frequency $\omega$ is treated as a parameter and not determined self-consistently.

We conclude this Section with a remark concerning the behaviour of the trapped particles. According to Eq. (27), the perturbed distribution $\bar{h}_{T}^{(1,0)}$ introduces in the solution the magnetic toroidal precession of the trapped particles. In the next Section, we will discuss in more detail how this precession can interact with the magnetic island. We anticipate that the $\bar{h}_{T}^{(1,0)}$ found above 
represents an asymptotic limit for the solution we will find in Section 5 if one supposes $\omega \gg \omega_{\mathrm{tp}}$.

\section{The $\omega \sim \omega_{D}$ Regime}

\subsection{Solution of the Drift-Kinetic Equation}

We now order the terms of Eq.(10) supposing $\omega \sim \omega_{D}$ (cf. Eq. (14)). As previously stated, our goal is to study the physical mechanisms linked to the mutual interaction between the island propagation and the precession of trapped particles around it. The ordering of the terms in Eq. (10) becomes

$$
\Delta \delta: 1: \Delta: \Delta \delta: \Delta \delta: \delta: \Delta \delta: \Delta \delta^{2}: \Delta^{2} \delta^{2}=\delta^{2}: \Delta \delta^{2}
$$

Again, as we did not include an adiabatic split of the Maxwellian, and as we are neglecting equilibrium gradients, we can set (see Section 4):

$$
g^{(0,0)}=0 .
$$

Let us then move to the $\mathcal{O}(\delta)$ equation. We have

$$
\frac{v_{\|}}{R q} \frac{\partial}{\partial \theta} g^{(1,0)}=0
$$

and this means

$$
g^{(1,0)}=\bar{h}_{P}^{(1,0)}+\bar{h}_{T}^{(1,0)} .
$$

The bar over a function again indicates that it is $\theta$-independent. We have again divided the solution in two parts, each describing a different region of the velocity space.

Next we write the $\mathcal{O}(\Delta)$ equation. This is simply

$$
\frac{v_{\|}}{R q} \frac{\partial}{\partial \theta} g^{(0,1)}=0
$$

So also $g^{(0,1)}=\bar{g}^{(0,1)}$ is $\theta$-independent.

The following step is to calculate the $\mathcal{O}\left(\delta^{2}\right)$ equation,

$$
\frac{v_{\|}}{R q} \frac{\partial}{\partial \theta} g^{(2,0)}+\frac{I v_{\|}}{R q} \frac{\partial}{\partial \theta}\left(\frac{v_{\|}}{\omega_{c}}\right) \frac{\partial}{\partial \chi} \bar{g}^{(1,0)}=-\frac{I v_{\|}}{R q} \frac{\partial}{\partial \theta}\left(\frac{v_{\|}}{\omega_{c}}\right) \frac{\partial \Phi}{\partial \chi} \frac{q_{i} F_{M}}{T}
$$

which can be integrated over $\theta$, so we have

$$
g^{(2,0)}=-I \frac{v_{\|}}{\omega_{c}} \frac{\partial}{\partial \chi}\left[\frac{q_{i} \Phi}{T} F_{M}+\bar{g}^{(1,0)}\right]+\bar{h}_{P}^{(2,0)}+\bar{h}_{T}^{(2,0)}
$$

where again we separate the trapped and passing contributions in defining the functions $\bar{h}_{P}^{(2,0)}$ and $\bar{h}_{T}^{(2,0)}$, that result as constants of integration. 
To evaluate the lowest-order contribution due to the island, i.e. the $\bar{g}^{(1,0)}$, we now turn to the $\mathcal{O}\left(\Delta \delta^{2}\right)$ equation, which has the form

$$
\begin{array}{r}
-\omega \frac{\partial}{\partial \xi} \bar{g}^{(1,0)}+\frac{v_{\|}}{R q} \frac{\partial}{\partial \theta} g^{(2,1)}+\left.k_{\|} v_{\|} \frac{\partial}{\partial \xi} \bar{g}^{(2,0)}\right|_{\Omega}+ \\
c \frac{\mathbf{B} \times \nabla \Phi}{B^{2}} \cdot \nabla \bar{g}^{(1,0)}+\frac{I v_{\|}}{R q} \frac{\partial}{\partial \theta}\left(\frac{v_{\|}}{\omega_{c}}\right) \frac{\partial}{\partial \chi} \bar{g}^{(1,1)}+ \\
-m \frac{I v_{\|}}{R q} \frac{\partial}{\partial \chi}\left(\frac{v_{\|}}{\omega_{c}}\right) \frac{\partial}{\partial \xi} \bar{g}^{(1,0)}=m \frac{I v_{\|}}{R q} \frac{\partial}{\partial \chi}\left(\frac{v_{\|}}{\omega_{c}}\right) \frac{\partial \Phi}{\partial \xi} \frac{q_{i} F_{M}}{T} .
\end{array}
$$

Let us solve this equation first in the trapped region of phase space. We use the bounce average operator defined for the trapped region (see Section 4). Recalling Eq. (38), this yields

$$
\begin{array}{r}
-\omega\left\langle\frac{R q}{\left|v_{\|}\right|}\right\rangle_{\theta}^{T} \frac{\partial}{\partial \xi} \bar{h}_{T}^{(1,0)}-I\left\langle\left. R q k_{\|} \frac{\partial}{\partial \xi}\left(\frac{\left|v_{\|}\right|}{\omega_{c}} \frac{\partial}{\partial \chi} \bar{h}_{T}^{(1,0)}\right)\right|_{\Omega}\right\rangle_{\theta}^{T}+ \\
+c\left\langle\frac{R q}{\left|v_{\|}\right|} \frac{\mathbf{B} \times \nabla \Phi}{B^{2}}\right\rangle_{\theta}^{T} \cdot \nabla \bar{h}_{T}^{(1,0)}-m I\left\langle\frac{\partial}{\partial \chi} \frac{\left|v_{\|}\right|}{\omega_{c}}\right\rangle_{\theta}^{T} \frac{\partial}{\partial \xi} \bar{h}_{T}^{(1,0)}= \\
m I\left\langle\frac{\partial}{\partial \chi} \frac{\left|v_{\|}\right|}{\omega_{c}}\right\rangle_{\theta}^{T} \frac{\partial \Phi}{\partial \xi} \frac{q_{i} F_{M}}{T}+I\left\langle\left. R q k_{\|} \frac{\partial}{\partial \xi}\left[\frac{\left|v_{\|}\right|}{\omega_{c}} \frac{\partial}{\partial \chi}\left(\frac{q_{i} \Phi}{T} F_{M}\right)\right]\right|_{\Omega}\right\rangle_{\theta}^{T} .
\end{array}
$$

We note that

$$
\left\langle\frac{\partial}{\partial \theta}\left(\frac{\left|v_{\|}\right|}{\omega_{c}}\right) \frac{\partial g^{(1,1)}}{\partial \chi}\right\rangle_{\theta}^{T}=0
$$

because, for symmetry reasons, we can suppose $g^{(1,1)}$ to be an even function in $\theta$. We also suppose that $\bar{h}_{T}^{(2,0)}$ is independent on $\sigma$, which is consistent with the bounce point continuity condition.

An analytic solution of Eq. (40) is extremely difficult. However, we can greatly simplify this equation by employing the same approximation as in Eq. (25) (for the details of the calculation see Appendix I). If we focus on the dynamics along the island (the radial component of the $\mathbf{E} \times \mathbf{B}$ drift, which goes to zero faster with $\chi$ than the other terms, will be shown later to be important only to unlock resonating particles), it is possible to write Eq. (40) as:

$$
\left[-\omega-\frac{m}{q} \omega_{E}-\frac{m}{q} \omega_{\mathrm{tp}}\right] \frac{\partial \bar{h}_{T}^{(1,0)}}{\partial \xi}=\frac{m}{q} \omega_{\mathrm{tp}} \frac{q_{i} F_{M}}{T} \frac{\partial \Phi}{\partial \xi} .
$$

Eq. (41) can be integrated with the condition $\bar{h}_{T}^{(1,0)} \rightarrow 0$ for $\chi \rightarrow \infty$. For the sake of simplicity, we neglect the dependence of $\omega_{E}$ on $\xi$, so that the only quantity depending on $\xi$ is the electrostatic potential. 


$$
\bar{h}_{T}^{(1,0)}=-\frac{m}{q} \frac{\omega_{\mathrm{tp}}}{\omega+\frac{m}{q} \omega_{E}+\frac{m}{q} \omega_{\mathrm{tp}}} \frac{q_{i} \Phi}{T} F_{M} .
$$

Note that in this case the $\chi$ part of the potential plays the role of an integration constant. The physical implications of Eq.(42) will be discussed in the following Section.

We now turn back to Eq.(39) and we solve it in the passing region of phase space. Again we suppose that the bounce average operator (see Section 4) cancels the $g^{(1,1)}$ contribution. The fundamental difference with respect to the trapped particles is that for passing particles

$$
\left\langle\frac{\partial}{\partial \chi}\left(\frac{v_{\|}}{\omega_{c}}\right)\right\rangle_{\theta}=\mathcal{O}\left(\epsilon^{2}\right)
$$

so the term on the right-hand side of Eq. (39) is negligible. This equation becomes then

$$
-\omega \frac{\partial}{\partial \xi} \bar{h}_{P}^{(1,0)}-\left\langle\left. R q k_{\|} \frac{\partial}{\partial \xi} g^{(2,0)}\right|_{\Omega}\right\rangle_{\theta}+c \frac{\mathbf{B} \times \nabla \Phi}{B^{2}} \cdot \nabla \bar{h}_{P}^{(1,0)}=0 .
$$

We can choose

$$
\bar{h}_{P}^{(1,0)}=0 .
$$

This solution is consistent with the fact that, in this regime, the contribution of the passing particles to the perpendicular current is negligible. So we obtain

$$
\left\langle\left. R q k_{\|} \frac{\partial}{\partial \xi} g^{(2,0)}\right|_{\Omega}\right\rangle_{\theta}=0
$$

which in view of Eq. (38) leads to

$$
\bar{h}_{P}^{(2,0)}=\left\langle I \frac{v_{\|}}{\omega_{c}} \frac{\partial}{\partial \chi} \frac{q_{i} \Phi}{T} F_{M}\right\rangle_{\theta} .
$$

Inserting Eq. (45) into Eq. (38), we see that these equations are linked to the annihilation in the passing region of the phase space discussed in Section 4. Within the ordering employed in this Section, this contribution still exists, but it pertains to a higher order because of the island propagation frequency ordering, and by consequence all the purely electric effects (see Eq.(7)) become less important.

\subsection{The Perturbed Current}

The velocity perpendicular to perturbed magnetic surface can be thought as the variation of the $\Omega$ coordinate with respect to time, i.e. $d \Omega / d t$. We are interested in the lowest-order, $\theta$-averaged perturbed current.

By definition of total derivative, and noticing that $\nabla_{\|} \Omega=0$, we can write 


$$
\frac{\mathrm{d} \Omega}{\mathrm{d} t}=\frac{\partial \Omega}{\partial t}+\mathbf{v} \cdot \nabla \Omega=\frac{\partial \Omega}{\partial t}+\left(\mathbf{v}_{\mathbf{E} \times \mathbf{B}}+\mathbf{v}_{\mathbf{D}}\right) \cdot \nabla \Omega .
$$

Considering Eq.(7), and recalling that

$$
\frac{\partial}{\partial t}=-\omega \frac{\partial}{\partial \xi}
$$

we note that

$$
\frac{\partial \Omega}{\partial t}+\mathbf{v}_{\mathbf{E} \times \mathbf{B}} \cdot \nabla \Omega=0
$$

and this means, for trapped particles

$$
\frac{\mathrm{d} \Omega}{\mathrm{d} t}=-\frac{m}{q} \omega_{D} \sin \xi
$$

The radial component of the magnetic drift does not contribute to the current we are interested in because it $\theta$-averages to zero even in the trapped region of phase space. So the toroidal precession in this island propagation frequency regime is the main mechanism which allows a particle to explore different magnetic surfaces. This is a fundamental difference with the previous Section, because for passing particles this contribution averages to $\mathcal{O}\left(\epsilon^{2}\right)$, so that what forces particles to explore different magnetic surface is not an equilibrium velocity contribution.

Eq. (47) allows us to write an approximate expression for the $\theta$-averaged current crossing the perturbed magnetic surface in presence of a slowly rotating NTM as a function of $v$ :

$$
J_{\perp}(v)=q_{i}\left\langle g^{(1,0)} \frac{\mathrm{d} \Omega}{\mathrm{d} t} \frac{1}{|\nabla \Omega|}\right\rangle_{\theta}=\frac{m^{2}}{q^{2}} \frac{q_{i}^{2} \Phi}{T} \frac{\omega_{D} \omega_{\mathrm{tp}}}{\omega+\frac{m}{q} \omega_{\mathrm{tp}}+\frac{m}{q} \omega_{E}} \frac{1}{|\nabla \Omega|} F_{M} \sin \xi
$$

estimating with $\mathrm{d} \Omega / \mathrm{d} t \cdot 1 /|\nabla \Omega|$ the velocity component perpendicular to the perturbed magnetic surface. We remind that $g^{(1,0)}$ is nonzero only for trapped particles. This expression for the perpendicular current at low rotation frequencies is confirmed by our numerical simulations.

We see in Fig. 3 that for positive frequencies no particle can be resonant with the NTM, since the island drift and the precession of the trapped particles are in opposite directions, so the distribution is quite smooth. On the other hand, if the frequency is negative, the distribution starts to be very peaked around that region of phase space where $\omega \approx n \omega_{\text {tp }}$ (we recall that $\omega_{\text {tp }}$ depends on $v$ ), and changes sign around this critical value of velocity.

Another important information which can be gained from Fig. 3 is that, for the trapped-particle resonance under consideration, the physical effect that resolves the singularity in Eq. (48) is represented by collisions, even for the low-collisionality regime discussed in this paper. This is consistent with the 
(a)

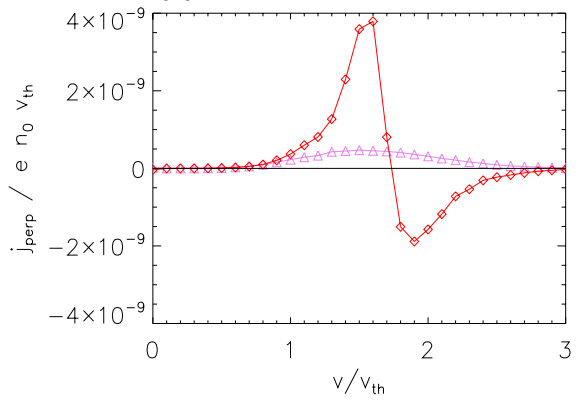

(b)

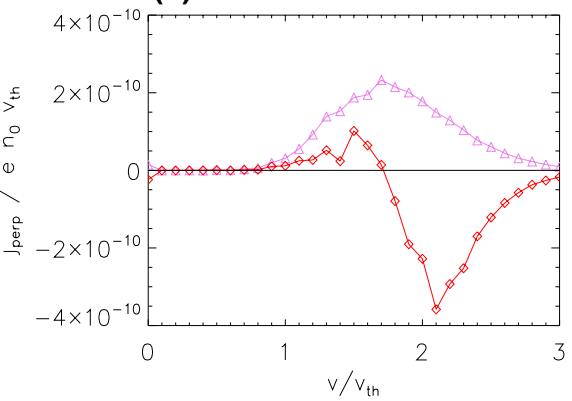

Figure 3: Perpendicular current as a function of velocity, calculated at the fourth radial cells outside the magnetic island towards the plasma centre, for $\omega=300 \mathrm{rad} / \mathrm{s}$ (triangles) and for $\omega=-300 \mathrm{rad} / \mathrm{s}$ (diamonds) in the collisionless regime (a) and in a standard banana collisional regime (b). Note the different scale on the $y$-axis.

fact that, in our simulations, the frequency on which trapped particles can be scattered into the passing domain is comparable or higher than the toroidal precession of thermal ions $\omega_{\mathrm{tp}} \sim \omega$. Fig. $3 \mathrm{~b}$ is obtained in the standard banana regime $\left(\nu_{\text {coll }} / \epsilon \sim 10^{2} \mathrm{~Hz}\right.$, and $\omega_{b} / 2 \pi \sim 10^{4} \mathrm{~Hz}$, where $\nu_{\text {coll }}$ is the collision frequency and $\omega_{b}$ the banana bounce frequency), whereas in Fig. 3a, where the collision frequency is reduced by five orders of magnitude (we call this a "collisionless" regime). Collisions drastically reduce the peaks of $J_{\perp}(v)$ around the resonance, this effect being more pronounced for slower particles, as can be expected. As a consequence of this last fact, in particular, the sign of the total perpendicular current density (i. e. of the integral of $J_{\perp}(v)$ over $v$ ) can change depending on the collision frequency, as shown in Fig. 4a. The sign of the perpendicular current is of course crucial for the determination of the stabilizing or destabilizing nature of the perturbed parallel current.

To evaluate explicitely the influence of this current on the NTM stabilization, we need to compute the closure parallel current. However, the integration over the velocity space cannot be performed because of the resonant denominator in $\bar{h}_{T}^{(1,0)}$ (see Eq. (42)). To account for the effect of collisions, we go back to Eq. (41) and we add a simple Krook collision operator $\left(\partial f /\left.\partial t\right|_{\text {coll }}=-\nu\left(f-F_{M}\right)\right.$, where $\left(f-F_{M}\right)=\bar{h}_{T}^{(1,0)}$ and $\left.\nu=\nu_{0} v_{\mathrm{th}}^{3} / v^{3}\right)$. We obtain

$$
\left[-\omega-\frac{m}{q} \omega_{\mathrm{tp}}-\frac{m}{q} \omega_{E}\right] \frac{\partial \bar{h}_{T}^{(1,0)}}{\partial \xi}=\frac{m}{q} \omega_{\mathrm{tp}} \frac{q_{i} F_{M}}{T} \frac{\partial \Phi}{\partial \xi}-\nu \bar{h}_{T}^{(1,0)},
$$

Again, we neglect the $\xi$ dependence of $\omega_{E}$ for the sake of simplicity. Indicating

$$
\bar{\omega}=\omega+\frac{m}{q} \omega_{\text {tp }}+\frac{m}{q} \omega_{E} \quad \bar{F}=\frac{m}{q} \omega_{\text {tp }} \frac{q_{i} F_{M}}{T}
$$



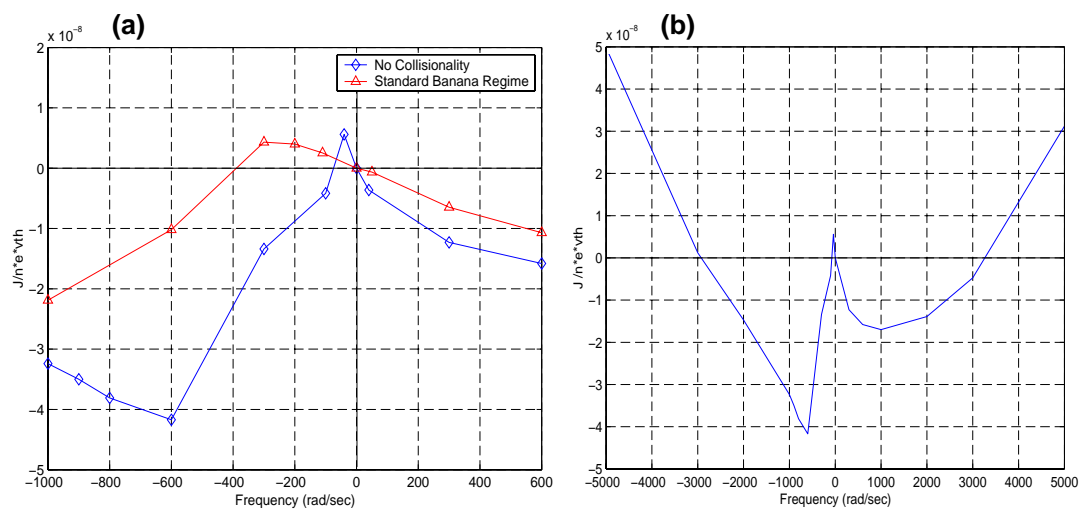

Figure 4: a) Comparison between the perpendicular current integrated in the velocity space versus island propagation frequency in non collisional regime (diamonds) and in standard banana regime (triangles).b) Perpendicular current integrated in the velocity space versus island propagation frequency in the non-collisional regime showing the transition to the standard polarization current (proportional to $\omega^{2}$ ) at high frequencies.

and expanding the island potential into its Fourier components

$$
\Phi=\sum_{k=0}^{\infty} \hat{\Phi}_{k}(\chi) \cos (k \xi)
$$

a solution to Eq. (49) is found in the form

$$
\bar{h}_{T}^{(1,0)}=-\bar{\omega} \bar{F} \sum_{k=0}^{\infty} \frac{k^{2} \hat{\Phi}_{k}(\chi)}{\bar{\omega}^{2} k^{2}+\nu^{2}} e^{\nu / \bar{\omega} \cdot \xi}-e^{\nu / \bar{\omega} \cdot \xi} \frac{\bar{F}}{\bar{\omega}} \int_{0}^{\xi} \mathrm{d} \xi^{\prime} e^{-\nu / \bar{\omega} \cdot \xi^{\prime}} \frac{\partial \Phi}{\partial \xi^{\prime}},
$$

using as a boundary condition the fact that the solution must be finite for $\bar{\omega} \rightarrow 0$.

Now we use the quasi-neutrality condition $\nabla \cdot \mathbf{J}=0$, multiplying the ion and electron drift-kinetic equations times the respective charge, and then summing them, neglecting the magnetic drifts of the electrons (cf. Ref. [11]). The $\theta-$ averaged parallel current is then

$$
\left.k_{\|} \frac{\partial J_{\|}}{\partial \xi}\right|_{\Omega}=m q_{i}\left\langle\int \mathrm{d}^{3} v \frac{I v_{\|}}{R q} \frac{\partial}{\partial \chi}\left(\frac{v_{\|}}{\omega_{c}}\right) \frac{\partial \bar{h}_{T}^{(1,0)}}{\partial \xi}\right\rangle_{\theta} \approx n q_{i} \int \mathrm{d}^{3} v \omega_{D} \frac{\partial \bar{h}_{T}^{(1,0)}}{\partial \xi}
$$

The approximations leading to the appearance of $\omega_{D}$ in the last step have been discussed previously (see again Appendix I for details). Exploiting Eq. (50), one obtains after some algebra

$$
\frac{\partial \bar{h}_{T}^{(1,0)}}{\partial \xi}=\bar{F} \sum_{k=0}^{\infty} k^{2} \hat{\Phi}_{k} \frac{\bar{\omega} k \sin (k \xi)-\nu \cos (k \xi)}{\bar{\omega}^{2} k^{2}+\nu^{2}} .
$$


We neglect the cosine terms in Eq. (52), because they are related to out-ofphase current contributions which are not involved in the island stabilization [2] (they contribute to determining the island rotation frequency; a detailed analysis of these effects, however, is beyond the scope of this paper). With this approximation

$$
\frac{\partial \bar{h}_{T}^{(1,0)}}{\partial \xi}=\bar{F} \sum_{k=0}^{\infty} \hat{\Phi}_{k} \frac{\bar{\omega} k \sin (k \xi)}{\bar{\omega}^{2}+\nu^{2} / k^{2}}=-\frac{\bar{\omega} \bar{F}}{\bar{\omega}^{2}+\nu_{\mathrm{eff}}^{2}} \frac{\partial \Phi}{\partial \xi},
$$

where the effective collision frequency $\nu_{\text {eff }}$ is implicitely defined by this equation.

Going back to Eq. (51), with the help of the pitch-angle variables $(v, \lambda)$ and supposing $\omega_{\mathrm{tp}}=\omega_{\mathrm{tp}}(v)$ (which relies on the fact that in the trapped region of phase space $\left.v \approx v_{\perp} \gg v_{\|}\right)$, one obtains

$$
J_{\|}=-n_{0} \frac{4}{\sqrt{\pi} k_{\|}} \sqrt{\epsilon} n^{2} \frac{q_{i}^{2}}{T} \omega_{\mathrm{tp}}^{0} \omega_{D}^{0} \frac{q}{m c} \frac{\mathrm{d} h}{\mathrm{~d} \Omega} K_{1}(\omega)\left[\cos (\xi)-\langle\cos (\xi)\rangle_{\Omega}\right],
$$

where

$$
K_{1}(\omega)=\int_{0}^{\infty} \mathrm{d} y y^{12} \frac{\omega e^{-y^{2}}\left(\omega+n \omega_{E}+n \omega_{\mathrm{tp}}^{0} y^{2}\right)}{\left(\omega+n \omega_{E}+n \omega_{\mathrm{tp}}^{0} y^{2}\right)^{2} y^{6}+\left(\nu_{\mathrm{eff}}^{0}\right)^{2}},
$$

having defined $y=v / v_{\mathrm{th}}$ and $\omega_{\mathrm{tp}}=\omega_{\mathrm{tp}}^{0} y^{2}, \nu_{\mathrm{eff}}=\nu_{\mathrm{eff}}^{0} / y^{3}$. The parameter $K_{1}(\omega)$ can be computed if all the plasma parameters are known, and it is important to note that it is the only factor in Eq. (54) which depends on the island rotation frequency. The integration has been performed within the condition that this parallel current vanishes when flux-surface averaged. From here on, we call this perturbed current the precessional current, and we indicate it as $J_{\|}^{\mathrm{Pr}}$. Its contribution to the island stability can be found with the help of the Ampère equation [2]:

$$
\sum_{ \pm} \int_{-1}^{\infty} \mathrm{d} \Omega \oint \mathrm{d} \xi \frac{J_{\|}^{\operatorname{Pr}} \cos (\xi)}{\sqrt{\Omega+\cos (\xi)}}=\frac{c}{8 \sqrt{2}} \Delta_{\operatorname{Pr}}^{\prime} \frac{w B}{R q}
$$

Here, the sum is defined over the $\chi>\chi_{s}$ and $\chi<\chi_{s}$ regions. It yields, after some algebra

$$
\Delta_{\mathrm{Pr}}^{\prime}=\frac{32 n_{0}}{\sqrt{2 \pi} w B} \sqrt{\epsilon} n^{2} \frac{q_{i}^{2}}{T} \omega_{\mathrm{tp}}^{0} \omega_{D}^{0} \frac{q}{m^{2} c^{2}} \frac{q_{s}}{q_{s}^{\prime}} R^{2} q^{2} K_{1}(\omega) K_{2},
$$

where $K_{2}$ is a negative constant defined as

$$
K_{2}=\int_{1}^{\infty} \frac{\mathrm{d} \Omega}{\sqrt{\Omega}} \oint \mathrm{d} \xi \frac{\cos ^{2}(\xi)-\cos (\xi)\langle\cos (\xi)\rangle_{\Omega}}{\Omega+\cos (\xi)} \simeq-6.65 .
$$

The sign of $\Delta_{\operatorname{Pr}}^{\prime}$ depends only on the sign of $K_{1}(\omega)$. We recall that positive values of $\Delta^{\prime}$ correspond to destabilizing effects [2]. Thus, we have a stabilizing current for $\omega>0$ and, if $\omega<0$, for $|\omega|$ sufficiently larger than $\omega_{\mathrm{tp}}$. 
(a)

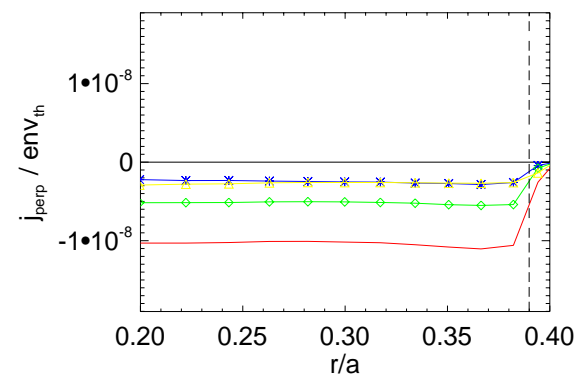

(b)

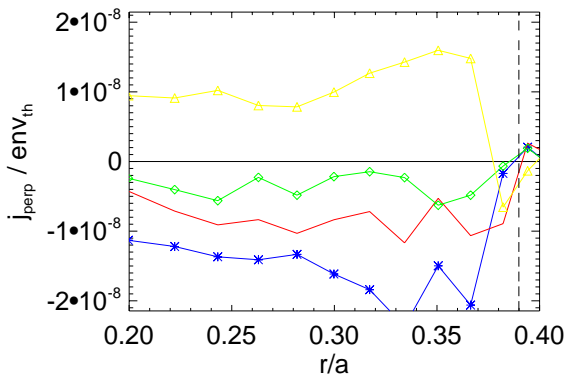

Figure 5: Current on the X-point helical cell (triangles), on the O-point cell (stars), on the intermediate cell (diamonds) and their sum (solid) for a) $\omega=300 \mathrm{rad} / \mathrm{s}$ and b) $\omega=-300 \mathrm{rad} / \mathrm{s}$.

We now compare the contribution we just found and that of the polarization current, in order to understand under which circumstances one prevails on the other. For the parallel current which closes the polarization current, Eq. (30), we refer to the $\omega \gg k_{\|} v_{\|}$case, so that $\bar{h}_{T}$ is zero, while for this comparison we refer to the parallel precessional current as expressed in Eq. (51). As we are comparing the two contributions in trapped space, we do not consider $\bar{h}_{P}$ functions. Using the identity Eq. (62), see Appendix I, we can write

$$
\left.k_{\|} \frac{\partial J_{\|}^{\mathrm{Pol}}}{\partial \xi}\right|_{\Omega}=-\frac{q_{i} I^{2}}{\omega_{c}^{2}} \frac{\omega^{2} q}{m c} \frac{d h}{d \Omega} \frac{8}{W_{\chi}^{2}} \int \mathrm{d}^{3} v v_{\|}^{2} \frac{\partial}{\partial \chi}\left(\frac{d h}{d \Omega}\right) \frac{q_{i} F_{M}}{T} \sin \xi .
$$

We can compare $J_{\|}^{\mathrm{Pr}}$ and $J_{\|}^{\mathrm{Pol}}$ by comparing the two functions under the integration operator. So, the two contribution are comparable if

$$
\frac{I}{\omega_{c}^{2}} \omega \frac{1}{W_{\chi}^{2}} \frac{\partial}{\partial \chi} \frac{d h}{d \Omega} \sim \frac{m}{R q} \frac{\partial}{\partial \chi}\left(\frac{v_{\|}}{\omega_{c}}\right) \frac{n \omega_{\mathrm{tp}}}{\omega+n \omega_{\mathrm{tp}}},
$$

where $\omega_{E}$ in Eq. (51) has been discarded for the sake of simplicity. If we are not so far away from the island (i.e. $\left.\left(\chi-\chi_{s}\right) \sim W_{\chi}\right)$ the derivative in $\chi$ of $\mathrm{d} h / \mathrm{d} \Omega$ is $\mathcal{O}(1)$. According to the estimate (see Appendix I)

$$
\frac{\partial}{\partial \chi}\left(\frac{v_{\|}}{\omega_{c}}\right) \approx \frac{R q \omega_{\mathrm{tp}}}{I v_{\|}}
$$

Eq. (58) becomes

$$
\frac{I^{2}}{\omega_{c}^{2}} \omega \frac{v_{\|}^{2}}{W_{\chi}^{2}} \sim n \omega_{\mathrm{tp}} \frac{n \omega_{\mathrm{tp}}}{\omega} .
$$

We estimate $v_{\|} \sim \sqrt{\epsilon} v_{\text {th }}$. Recalling that the ion banana width $\rho_{b}$ can be calculated as $\rho_{b}=\sqrt{\epsilon} \rho_{\theta}$, we can conclude that the polarization current is comparable with the precessional current if 


$$
\frac{n \omega_{\mathrm{tp}}}{\omega} \sim \frac{\rho_{b}}{w} .
$$

The ratio on the right-hand side of Eq. (61) is assumed to be small in our calculations, cf. Eq.(13), so that the assumption that the ratio on the left-hand side of Eq. (61) is of the same order of magnitude is absolutely realistic.

\section{Discussion and Conclusions}

We have studied the plasma current due to a rotating magnetic island when the island propagation frequency is comparable to the parallel streaming of the passing particles $\left(k_{\|} v_{\|}\right)$or to the toroidal precession frequency the trapped particles $\left(\omega_{\mathrm{tp}}\right)$ in a low-collisional plasma. When the absolute value of $\omega$ is large $\left(\omega \gg k_{\|} v_{\|}\right.$, assuming for passing particles $\left.v_{\|} \sim v_{\text {th }}\right)$, the polarization current, which scales like $\omega^{2}$ in absence of equilibrium gradients, is the dominant contribution. In Section 4, we showed that even if $\omega \sim k_{\|} v_{\|}$the polarization current remains the most important perturbed current linked to the presence of the island rotation, since the contribution of the resonating (passing) particles, significant for the distribution function, is almost cancelled when the corresponding current is calculated. In Section 5, we showed that for decreasing values of $\omega$ other electric and magnetic effects dominate over the polarization current. In particular, the toroidal electric field generated by a magnetic island can modify the magnetic toroidal precession a trapped particle experiences in an equilibrium configuration, braking or accelerating the particle itself. This leads to the appearance of a precessional current competing with the polarization current. For a more detailed physical explanation of this effect, we distinguish between positive and negative frequencies. For positive frequencies, the magnetic island is moving towards $-\nabla \zeta$-direction, while both electric and magnetic toroidal drifts point in the $\nabla \zeta$-direction. If we suppose to build a frame of reference which moves in the toroidal direction together with the island (from here on: IFR, island frame of reference), we will see all the trapped particles travelling in the $\nabla \zeta$-direction. The toroidal component of the electric field $E_{\zeta}$ varies sinusoidally along the island, see Eq. (7). So in regions where it points in the $\nabla \zeta$-direction, all particles tend to increase their kinetic energy and finally their magnetic precession frequency (recall $\omega_{\text {tp }} \propto v^{2}$ ), while they slow down in the opposite case. This means that between $\mathrm{O}-\mathrm{X}$ and between $\mathrm{X}-\mathrm{O}$, all trapped particles either accelerate or decelerate, depending on the sign of $E_{\zeta}$ (they accelerate for $E_{\zeta}>0$ and decelerate for $E_{\zeta}<0$, respectively). Where they decelerate, they tend to accumulate, so that the local density increases. On the contrary, they tend to disperse as they accelerate, so that the local density decreases. Fig. 5a) shows this situation in the "upper" half of the island (as stated before, the situation is opposite in the "lower" half).

This picture is different when $\omega<0$. In this case the island is propagating in the same direction as the particles ( $\nabla \zeta$-direction), so in the IFR there are particles moving in the $\nabla \zeta$ direction (if $|\omega|<\omega_{\text {tp }}$, high-energy particles) 
and in the $-\nabla \zeta$ direction (if $|\omega|>\omega_{\mathrm{tp}}$, low-energy particles). Now, when for example $E_{\zeta}$ points in the $-\nabla \zeta$-direction, again all particles decrease their magnetic precession frequency. The behaviour of more energetic particles is the same as the one described before. But slower particles, if decelerated in the laboratory frame, actually increase their relative speed with respect to the island, so the effect is an acceleration in the IFR.In other words, where slower particles accumulate, faster particle disperse and viceversa, and this explains why the perturbation changes sing around $\omega_{\mathrm{tp}} \sim|\omega|$. Moreover, another mechanism complicates the overall picture in the case $\omega<0$. In this case, the electric toroidal precession $\omega_{E}$ acts in the opposite direction with respect to the magnetic drifts. So, moving from O-point (where the radial electric field is maximum, in absolute value) to X-point (where the radial electric field is the lowest in absolute value) the number of particles which overtake the island or are overtaken by it in IFR can change (cf. Eq.(42)). The variation of $\omega_{E}$ with $\xi$ is such that the integral of $J_{\perp}$ over velocity space can change its sign depending on where with respect to the island we are performing the integration. This especially happens if $|\omega| \sim \omega_{\mathrm{tp}}\left(v_{\mathrm{th}}\right)$, because in that region of phase space lies a large number of particles, so even a small shift of the resonant point means turning a large number of faster particles into slower or vice versa (cf. Fig. 3a)). This physical picture is confirmed in Fig. 5b). In this case, we are able to identify the change of sign of the current going from O-point to X-point, as indeed $|\omega| \approx \omega_{\mathrm{tp}}\left(v_{\mathrm{th}}\right)$. It is important to stress that resonance conditions are highly local, so after a while a resonant particle will be able to unlock from the island, for example through the radial component of the $\mathbf{E} \times \mathbf{B}$ drift.

There are some strong analogies between the behaviour of the trapped and passing particles as their motion along the island start to be comparable with the rotation of the island itself (i.e. the case $\omega \sim \omega_{D}$ and $\omega \sim k_{\|} v_{\|}$, respectively). In both cases, the lowest-order perturbed distribution function exhibits a resonant denominator (Eq. (23) and (42)), which underlines the fact that the interaction between the particles and the mode (and the subsequent modification of the distribution function) is stronger if the particle and the island have a small relative motion. Indeed, this result is not surprising for most waveparticle interactions. Nevertheless, significant differences occur as we focus on the corresponding perturbed current. Trapped particles cross the perturbed magnetic surfaces just because of their equilibrium drift velocity (see Eq.(47)), so that every perturbation on the distribution immediately leads to a current such that

$$
\delta J_{\perp} \propto q_{i}\left\langle\delta f \mathbf{v}_{D} \cdot \nabla \Omega\right\rangle_{\theta} .
$$

This is not the case for passing particles, as their $\theta$-averaged equilibrium drift across the perturbed flux-surface is much smaller. For resonating particles, in particular, the advecion is such that it nearly cancels the contribution of the perturbed distribution function to the current, as shown in Section 4.

Referring to Fig. 4b), we can now discuss all the changes of sign of $J_{\perp}$ as a function of $\omega$, going from right to left. For positive island frequencies, we 
experience a change of sign when the precessional current starts to exceed the standard polarization current. The sign reversal at $\omega=0$ is due to the fact that the electric potential goes through zero and changes sign across that value (cf. Eq. (7) and Eq. (42)). As a matter of fact, for extremely small negative values of $\omega$, the situation more or less corresponds to the one which occurs for small positive values of $\omega$, as almost all particles are faster than the island. So the sign reversal is due to a sign reversal in the electric field. As $\omega$ grows, the fraction of slower particles gets larger, and this leads to the third change of sign. Collisions contribute to determine the position of this third reversal, since they determine how the singularity in Eq. (48) is resolved. Finally, for large negative values of $\omega$ the polarization current prevails again. The qualitative agreement between the analytical and numerical results is remarkable.

The change of sign determines the stabilizing or destabilizing effect of these currents. The precessional current is found to be stabilizing for $\omega>0$ and, if $\omega<0$, for $|\omega|$ sufficiently larger than $\omega_{\mathrm{tp}}$. It is known that polarization current is globally destabilizing, neglecting equilibrium gradients effects, because of the "current spike" at the island separatrix [30], without which it would be stabilizing. This precessional current acts against the polarization current (which is in our case always destabilizing, according to the analysis of Ref. [30]). We emphasize that in this paper we neglected effects connected to equilibrium pressure gradients, as our aim was not a complete determination of the island dynamics, but rather the discussion of the contributions linked to the island rotation and to possible resonances with the motion of the particles. Our finding that precessional effects can compete with the neoclassical polarization and that trapped-particle resonances have a major impact on this effect highlights once again that a kinetic description is mandatory in view of a exhaustive theory of NTMs in toroidal plasmas.

\section{Appendix I}

In this Appendix the relation between the toroidal magnetic precession frequencies of trapped particles $\omega_{D}$ and $\omega_{\hat{s}}$ and the corresponding terms in Eq. (41) is briefly discussed. We start wit $\omega_{D}$, which deals with the poloidal component of the equilibrium magnetic drift $\mathbf{v}_{D}$. As the spatial derivatives have to be taken at constant kinetic energy, one can write [21]

$$
\nabla v_{\|}=-\frac{1}{m_{i} v_{\|}} \mu \nabla B .
$$

Using this relation, recalling that the parallel velocity and the cyclotron frequency depend on space only through the magnitude of the magnetic field, one can write

$$
\frac{\partial}{\partial \chi}\left(\frac{v_{\|}}{\omega_{c}}\right)=-\frac{1}{\omega_{c}}\left[\frac{\mu}{m_{i} v_{\|}}+\frac{v_{\|}}{B}\right] \frac{\partial B}{\partial \chi} .
$$


In the large aspect ratio approximation $B=B_{0}(1-\epsilon \cos \theta)$, so

$$
\frac{\partial B}{\partial \chi}=-\frac{q}{r R} \cos \theta
$$

neglecting higher order terms in $\epsilon$. This implies

$$
\frac{\partial}{\partial \chi}\left(\frac{v_{\|}}{\omega_{c}}\right)=\frac{q}{r R \omega_{c}}\left[\frac{\mu}{m v_{\|}}+\frac{v_{\|}}{B}\right] \cos \theta
$$

The poloidal component of the magnetic drift can for this reason be written as

$$
m \frac{I v_{\|}}{R q} \frac{\partial}{\partial \chi}\left(\frac{v_{\|}}{\omega_{c}}\right)=\frac{m}{q} \frac{q}{r R \omega_{c}}\left[\frac{\mu B}{m_{i}}+v_{\|}^{2}\right] \cos \theta .
$$

This result, after being bounce averaged, corresponds exactly to $(m / q) \omega_{D}$ (see Eq. (14)).

We now focus on the relation between the terms in $k_{\|} v_{\|}$in the Eq. (40) and the magnetic shear frequency $\omega_{\hat{s}}$. We concentrate on

$$
\left.I \frac{v_{\|}}{\omega_{c}} k_{\|} v_{\|} \frac{\partial}{\partial \xi}\right|_{\Omega}\left(\frac{\partial}{\partial \chi} \Phi\right)
$$

because all terms related to $\omega_{\hat{s}}$ have this form in our equations. It is easy to see that

$$
\left.I \frac{v_{\|}}{\omega_{c}} k_{\|} v_{\|} \frac{\partial}{\partial \xi}\right|_{\Omega}\left(\frac{\partial \Phi}{\partial \chi}\right)=-\left.I \frac{v_{\|}}{\omega_{c}} k_{\|} v_{\|} \frac{4}{W_{\chi}^{2}} \frac{\omega q}{m c} \frac{d h}{d \Omega} \frac{\partial \chi}{\partial \xi}\right|_{\Omega} .
$$

Recalling that

$$
\left.k_{\|} \frac{\partial \chi}{\partial \xi}\right|_{\Omega}=\frac{m}{q} \frac{\partial A_{\|}}{\partial \xi}=\frac{m}{q} \frac{\tilde{\psi}}{R} \sin \xi
$$

and

$$
\tilde{\psi}=\frac{W_{\chi}^{2}}{4} \frac{q_{s}^{\prime}}{q_{s}}
$$

one can find with a few algebra

$$
\left.I \frac{v_{\|}}{\omega_{c}} k_{\|} v_{\|} \frac{\partial}{\partial \xi}\right|_{\Omega} \frac{\partial \Phi}{\partial \chi}=\frac{m}{q} \frac{q \hat{s} v_{\|}^{2}}{r^{2} \omega_{c}} \frac{\partial \Phi}{\partial \xi},
$$

where $\hat{s}$ is the magnetic shear $(\hat{s}=r / q \mathrm{~d} q / \mathrm{d} r)$ and the shear precession frequency [22] can be easily identified after $\theta$-averaging this expression. 


\section{References}

[1] H. P. Furth, J. Killeen, M. N. Rosenbluth Phys. Fluids 6459 (1963)

[2] P. H. Rutherford, Phys. Fluids 161903 (1973)

[3] R. Carrera, R. D. Hazeltine, M. Kotschenreuther, Phys. Fluids 29899 (1986)

[4] W. X. Qu and J. D. Callen University of Wisconsin Plasma Report No UWPR 85-5 (1985)

[5] Z. Chang et al., Phys. Rev. Letters 744663 (1995)

[6] O. Sauter et al., Phys. Plasmas 41654 (1997)

[7] E. Poli et al., Phys. Rev. Letters 94205001 (2005)

[8] A. I. Smolyakov, Sov. J. Plasma Phys. 15, 667 (1989)

[9] A. B. Mikhailovskii, Contrib. Plasma Phys. 43, 125 (2003)

[10] A. I. Smolyakov et al., Phys. Plasmas 21581 (1995)

[11] H. R. Wilson et al., Phys. Plasmas 3248 (1996)

[12] A. B. Mikhailovskii, V. D. Pustovitov, A. I. Smolyakov, Plasma Phys. Control. Fusion 42, 309 (2000)

[13] A. Bergmann, E. Poli, A. G. Peeters, Phys. Plasmas 12072501 (2005)

[14] E. Poli et al., Nucl. Fusion 45384 (2005)

[15] S. V. Konovalov et al., Doklady Physics 47488 (2002)

[16] A. I. Smolyakov, Plasma Phys. Control. Fus. 35657 (1993)

[17] J. W. Connor, H. R. Wilson, Phys. Plasmas 2, 4575 (1995)

[18] F. L. Waelbroeck J. W. Connor, H. R.Wilson, Phys. Rev. Letters 87 215003-1 (2001)

[19] M. James, H. R. Wilson, Plasma Phys. Control. Fus 481647 (2006)

[20] S. D. Pinches et al., Comput. Phys. Commun. 111133 (1998)

[21] R. D. Hazeltine and J. D. Meiss, Plasma Confinement (Addison-Wesley, New York, 1992)

[22] A. G. Peeters, PhD Thesis, Technische Universiteit Eindhoven, unpublished (1994)

[23] A. Bergmann, A. G. Peeters, S. D. Pinches, Phys. Plasmas 85192 (2001) 
[24] E. Poli et al, Plasma Phys. Control. Fus. 4571 (2003)

[25] F. L. Hinton, J. A. Robertson, Phys.Fluids 27(5) 1243 (1984)

[26] H. L. Berk, B. N. Breizman, Phys Fluids B 2, 2226 (1990)

[27] H. L. Berk, B. N. Breizman, Phys Fluids B 2, 2235 (1990)

[28] H. L. Berk, B. N. Breizman, Phys Fluids B 2, 2246 (1990)

[29] V. S. Marchenko, Nucl. Fusion 39, 1541 (1999)

[30] F. L. Waelbroeck, R. Fitzpatrick, Phys. Rev. Letters 781703 (1997) 